\title{
Commercial Aspects of Microarray Technology
}

\author{
Ken Rubenstein \\ The Lion Consulting Group, San Mateo, CA, USA
}

BioTechniques 34:S52-S54 (March 2003)

\section{INTRODUCTION}

DNA microarrays have come to play a central role in the rapidly evolving field of transcriptomics. They also have an important, if not key, role to play in DNA resequencing for scoring mutations and SNPs (single nucleotide polymorphisms) in support of in vitro diagnostic studies and gene-disease association studies of genetically complex diseases. Furthermore, successes in using DNA microarrays have stimulated efforts to undertake the much more difficult and complex task of developing protein microarrays.

Aspiring microarray users are faced with numerous choices. DNA arrays can be generated to contain as few as 10 probes or as many as 500000 . They can be made using cDNAs or oligonucleotides as probes. Oligonucleotides can be either synthesized in situ or spotted onto the array surface. Oligonucleotides can range in length from 25- to 100-mers or even longer. Users can choose to use the same "genome-wide" array for a broad range of experiments, or they can opt for focused arrays containing just the genes of interest for a particular experiment.

Perhaps the most difficult choice of all for users is whether to buy DNA microarrays from a commercial source or have them made at their own institution. Many large universities, public research institutions, and some companies have established core microarray facilities capable of making spotted cDNA or oligonucleotide arrays. Soon, they will be offered the possibility of producing synthesized oligonucleotide arrays on site (see below). Alternatively, users can choose to buy arrays from any of several commercial sources.

\section{COMMERCIAL MICROARRAY SOURCES}

The first and still the most popular commercial source, Affymetrix (Santa Clara, CA, USA), uses photolithographic technology adapted from the semiconductor industry to produce a broad line of synthesized oligonucleotide arrays with up to 500000 features (each a discrete area containing multiple copies of a single oligonucleotide) representing more than 16000 genes. Although best known for supplying genome-wide arrays, Affymetrix has recently focused attention on its CustomExpress program, which allows users to order lower-priced custom-made arrays based on selecting combinations of up to 1000 genes already in the Affymetrix repertoire.
Other prominent commercial sources of DNA microarrays include Agilent Technologies (Wilmington, DE, USA), Amersham Biosciences (Piscataway, NJ, USA) (which recently acquired the Motorola Life Sciences' CodeLink array product line [Motorola Life Sciences, Pasadena, CA, USA]), BD Biosciences (San Jose, CA, USA), and Applied Biosystems (Foster City, CA, USA). Agilent, for example, provides cDNA arrays representing 12000 to about 15000 genes, and at least one oligonucleotide array containing 14800 60-mer features. Agilent also offers custom oligonucleotide arrays in a variety of configurations.

\section{ARRAY CUSTOMIZATION ISSUES}

Each of the companies mentioned in the last paragraph is fully capable of supplying both broad-spectrum and focused "offthe-shelf" arrays of adequate quality, and several of them also offer custom arrays. It may be noted, however, that the current mainstream processes used to make both synthesized and spotted arrays are less than ideal for cost-effective customization. In essence, each spot or feature on each array requires a discrete act of deposition or synthesis, both of which are subject to variation unless great pains are taken to exercise process controls. Both array types require significant destructive quality assurance testing to assure adequate product performance. Production controls and subsequent quality checking add to both the expense and time required for completion of the production process.

Production stringencies are also partly responsible for the high prices users must pay for high-density positional arrays. Prices of $\$ 400$ or more per commercial array are not uncommon. Although quality products continually flow out from shipping department doors, very little is said or written about the excruciating process development issues vendors face in optimizing their procedures or, for that matter, the ratio of shippable units to discards. Industry watchers are well aware that Affymetrix spent at least 4 years, after the marketing introduction, getting the quality under sufficient control to reduce customer dissatisfaction to acceptable levels.

\section{AN ALTERNATIVE: ENCODED BEAD ARRAYS}

Encoded bead arrays, such as those provided by Luminex (Austin, TX, USA) and in development at several other compa- 
nies (e.g., Illumina [San Diego, CA, USA] and Quantum Dot [Palo Alto, CA, USA]) provide an attractive alternative for array customization. Beads are commonly encoded with dyes and conjugated to DNA probes, so that the fluorescence spectrum of a given bead contains discrete bands for coding dyes and the target label. Although no theoretical limit exists to the number of codes that can be used, current encoded bead array technologies are limited to levels of multiplexing on the order of 100. Products capable of 1000 or more codes are expected to enter the market in the next 1 to 3 years.

Encoded bead arrays are more amenable to customization than their positional counterparts. An array of 100 oligonucleotides can be prepared using aliquots taken from 100 batches of beads prepared ahead of time. Consequently, variability among bead arrays resulting from DNA immobilization is much less likely to be problematic than for positional arrays, in which the linkage of each probe to each feature in each array is a discrete event. A further advantage, one that is especially useful in the context of protein microarrays, is that the immobilization chemistry and the bead surface microenvironment can be customized for each DNA fragment, antibody, or other macromolecule immobilized. Since proteins cover an extremely broad range in their physicochemical characteristics, this flexibility attribute can be considered extremely useful, if not essential. Although different monoclonal antibodies are less variable in properties than proteins in general, they are, arguably, more variable than oligonucleotides.

Despite these advantages, encoded bead arrays have several disadvantages. For example, using the Luminex technology requires that users purchase a dedicated flow-cytometric analyzer. Illumina's technology, which uses beads captured on fiber optic bundles, requires users to purchase a dedicated instrument capable of reading fluorescence emission through an optical fiber bundle. Other systems require microscope-based robotic imaging systems. None of the current or forthcoming commercial systems has been adapted to make use of the large installed based of confocal microscope-based array scanners.

Based on the foregoing considerations, array users, constrained in their ability to order new capital equipment, already in possession of a confocal microarray scanner requiring several hundred or more features per array, and dealing with relatively few samples per repetitive gene set, are apt to select 2-dimensional (2D) positional arrays; while users less sensitive to capital equipment purchase constraints and interested in analyzing large numbers of samples for a repetitive set of genes or SNPs may be better served opting for a bead-based array alternative. Those users in the substantial middle ground between these two extremes will have several interesting alternatives to consider in the coming years.

\section{ISSUES UNDERLYING THE 2D VS. BEAD ARRAY DICHOTOMY}

The 2D array vs. bead array dichotomy underlies another set of issues facing researchers. Standard 2D arrays are ideal for high levels of multiplexing (e.g., 5000 or more features), but less than ideal from the perspective of throughput. In this sense, $2 \mathrm{D}$ arrays are ideal for the early stages of research and development pro- grams, when researchers want to examine lots of genes to find which are expressed or shut off in some set of circumstances.

Once a gene subset has been identified, two issues must be faced. First is validation. For a variety of reasons, sets of expressed genes identified in different laboratories using different array sources or types may not be identical either in composition or degree of differential regulation. It has become customary to validate array results using quantitative real-time polymerase chain reaction (qRT-PCR) analysis, which has come to be trusted as a sort of gold standard. On the downside, qRT-PCR is not amenable to significant degrees of multiplexing, so that validation of more than a handful of genes requires running many separate data points.

The other issue facing 2D array users, after identifying a gene set of interest, comes when they decide to investigate the generality of their findings by assaying multiple samples, perhaps hundreds or even thousands of them. Standard 2D arrays are not amenable to processing in large batches, and each small batch of, say, 1-12 assays may require $24 \mathrm{~h}$ or more to process. While it is quite feasible to print arrays of 10-100 features in the wells of microtiter plates, no commonly used products in this category have yet reached the market (the company Genometrix, which is no longer in operation, used such plates on a service basis).

Bead-based arrays are much more amenable than their $2 \mathrm{D}$ cousins for high-throughput work. Their larger surface-to-area ratios improve hybridization kinetics, and aliquots of bead sets are readily dispensed to microplate wells. Products on the market or near to commercial introduction appear to be limited to degrees of multiplexing of 100 or fewer entities, whereas many programs require high-throughput analysis of gene sets between 100 and 500 in size.

\section{ALTERNATIVES TO AFFYMETRIX MICROARRAY PRODUCTION TECHNOLOGY}

On another front, Affymetrix is no longer alone in its home technology area. Several companies are developing technologies and products using digital light processors (DLPs) in place of photolithography for producing positional arrays based on in situ light-directed oligonucleotide synthesis. In producing its oligonucleotide arrays, Affymetrix uses photolithographic masks that direct light to array positions designated to accept the next base for chain elongation. DLPs contain hundreds of thousands of tiny mirrors, each of which can be rotated independently under software control. One can easily envision how these devices can be used to direct reflected light to desired positions of a $2 \mathrm{D}$ surface in a process analogous to that of Affymetrix.

One of the companies employing DLPs, Febit AG (Mannheim, Germany), is designing an instrument system, the Geniom ${ }^{\circledR}$, which not only allows users to design and build their own oligonucleotide arrays, but also to hybridize the target and complete the assay in one continuous real-time operation. The heart of the unit, the DNA Processor ${ }^{\mathrm{TM}}$, permits building arrays in a $2 \mathrm{D}$ chip containing a serpentine arrangement of microchannels. The user inputs target sequence information, and software determines the sequences and positions of required oligonucleotides, which are then synthesized within the channels. Each 
processor in the first version of the Geniom contains eight segments, which can be used as a single array for a single sample or eight smaller arrays for eight samples. After the array is prepared, a sample containing the labeled target is injected, hybridized, processed, and scanned using a charge-coupled device (CCD) camera. Assuming 64000 25-mers (the unit can also reliably synthesize 60-mers) are synthesized, the Geniom could generate two processors and assay up to 16 samples per day. The first version of this system is scheduled for launch in early 2003, and a second version, capable of dealing with up to 48 samples per day, is in development. Although the Geniom is expected to cost approximately $\$ 300000$, it may be noted that the genomic technology instrumentation market is characterized by deal-making flexibility, especially in the early stages after launching a new product.

The Febit system is unique in presenting users the opportunity to make an array, analyze it, and redesign, remake, and reanalyze it in real time. The company proposes to replace a batch-type research process with a sequential one. In a typical microarray process, the producer makes a batch of arrays to specification and distributes them to users who typically employ them for assays over an extended period of time. Generally, multiple users, each with their own scanner, will tap into the same batch of arrays. The Febit proposal offers users not only the opportunity to experiment with array design, but also to collect experimental results in real time. One potential disadvantage, aside from the large capital equipment expenditure, is that the synthesizer gets tied up with analysis, a circumstance that might be viewed as permitting flexibility at the cost of lowering overall productivity.

At least two other companies, Nimblegen (Madison, WI, USA) and Xeotron (Houston, TX, USA), are on the verge of challenging Affymetrix with DLP-based product and service offerings. Nimblegen, like Febit, uses Affymetrix-style photoprotection chemistry in its MAS ${ }^{\mathrm{TM}}$ system. Nimblegen is already offering customers the opportunity to order custom arrays with up to 195000 24-mers representing about 10000 genes. Starting in early 2003, Nimblegen plans to offer customers the opportunity to purchase the system and produce their own arrays.

Xeotron is taking a different approach to oligonucleotide synthesis. A microscale chip containing 4000 or 8000 etched chambers per square centimeter (corresponding to as many array features) serves as a matrix of reaction wells for standard oligonucleotide chemistry. The DLP directs light at selected chambers to generate an acid catalyst required for chain elongation. Xeotron plans to introduce a cancer-related array together with a custom microarray production service before the end of 2002. Because Xeotron uses standard synthesis chemistry, the company claims the capability to produce arrays containing 100 -mer or longer oligonucleotides.

Combimatrix (Mukilteo, WA, USA) is following a similar course to that of Xeotron, using an electrochemical rather than a light-directed process for in situ oligonucleotide synthesis based on conventional chemistry. Electrodes on a semiconductor chip program the production of arrays containing up to about 1000 features, each currently $100 \mu \mathrm{m}$ in diameter. Combimatrix, which believes that it can readily employ industry standard 0.25 $\mu \mathrm{m}$ semiconductor processing techniques to produce arrays with up to a million features in a square centimeter, plans initially to sell customers a hybridizer-scanner instrument plus software to aid in the design of arrays for production at the company. Com- bimatrix, which will later offer a desktop array synthesizer, has the commercial advantage of involvement in a marketing collaboration with Roche Diagnostics (Pleasanton, CA, USA).

The references to oligonucleotide length in preceding paragraphs underlie a still unresolved issue in the microarray research community concerning the optimal length of oligonucleotides for gene expression studies. Affymetrix-style oligonucleotide synthesis chemistry gives chain elongation yields in the low $90 \%$ range, while standard chemistry affords yields closer to $100 \%$. Lower yields translate to truncated chains, which require compensation in terms of feature redundancy and proliferation of control features. These realities translate to limitations of achievable chain length with photoprotection schemes.

Short oligonucleotide chains favor minimization of false positive results due to single base mismatched hybrids, while longer chains favor minimization of false positive results from "crossreactive" hybrid formation. Increased length also minimizes the number of oligonucleotides required to specify a single gene. Establishment of the ideal length is a complex issue that has yet to be resolved.

\section{MICROARRAY MARKET TRENDS}

Marketing is a key consideration for aspiring microarray companies. Affymetrix has managed to leverage its position as a technology pioneer offering a broad array of quality products to gain a worldwide market share estimated at greater than $75 \%$ in terms of revenues derived from the commercial sale of microarrays. The breadth and strength of Affymetrix's patent position has discouraged a number of companies with innovative technologies from entering the market, and some current aspirants for market share may ultimately be faced with the choice of taking an expensive license from Affymetrix or facing patent infringement litigation. For example, Qiagen's (Valencia, CA, USA) Operon Technologies subsidiary (Alameda, CA, USA), which once offered microarrays for sale, today limits its activities to providing ready-to-array oligonucleotide sets.

However, it is unlikely that Affymetrix will be able to fulfill all of the needs of microarray users. As mentioned earlier, users wishing to assay multiple samples for a small subset of genes may best be served by bead array technologies. A possible trend toward using arrays of 50-mer or longer oligonucleotides may favor technologies, such as Agilent's, which are based on conventional synthesis chemistry. Furthermore, the expense and inconvenience of using fluorescent labels for microarray assays have prompted several companies to start developing microarray products based on label-free biosensor technologies. The DNA microarray field remains highly dynamic from both technological and commercial perspectives, and the protein microarray field has barely begun to develop.

\section{Address correspondence to:}

Dr. Ken Rubenstein

The Lion Consulting Group

803 N. Humboldt St., Suite 204

San Mateo, CA 94401, USA

650-401-8582

rubenstein@sbcglobal.net 